\title{
Guidelines for clinical applications of spatio-temporal gait analysis in older adults
}

\author{
Reto W. Kressig and Olivier Beauchet, for the European GAITRite ${ }^{\circledR}$ Network Group \\ Department of Rehabilitation and Geriatrics, Geneva University Hospitals, Geneva-Thonex, Switzerland
}

\begin{abstract}
Quantifying spatio-temporal gait parameters in stationary and ambulatory geriatric settings may aid the early identification of potential fallers, as well as the documentation of illness-specific gait disorders and intervention-related changes in rehabilitative medicine. Bringing gait analysis out of the laboratory and into a clinical setting is the goal of the European GAITRite network group, initiated in 2003 in Geneva. To enhance reproducibility of gait measures and for better comparability of outcomes in clinical environments, a consensus on data collection was formulated and presented at the $2^{\text {nd }}$ European GAITRite Meeting in Marseilles. The guidelines presented here are intended to facilitate network collaborations and to provide guidance to clinicians who wish to implement spatio-temporal gait analysis in clinical settings.

(Aging Clin Exp Res 2006; 18: 174-176)

๑2006, Editrice Kurtis
\end{abstract}

\section{BACKGROUND}

Examining gait characteristics in older adults enhances our understanding of movement control in this population and helps to better target preventive interventions (1). For example, it has been shown that specific gait changes, such as increased gait variability and increased stride width, are reliable predictors of falls $(2,3)$. Increased gait variability during a simultaneous attention-demanding task also appears to be a particularly good marker of decline in gait control $(4,5)$. Clinicians have long sought to bring gait analysis out of the laboratory and into a clinical setting (6). However, attaining this goal has been hindered, mainly by the lack of appropriate measuring tools. Fortunately, the growing availability of commercialized, affordable and userfriendly spatio-temporal gait analysis systems now allows the transfer of these research findings into clinical routine. Quantifying spatio-temporal gait parameters in stationary and ambulatory geriatric settings may aid the early identification of potential fallers $(2,3)$ as well as the documentation of illness-specific gait disorders and intervention-related changes in rehabilitative medicine (7).

\section{EUROPEAN GAITRITE ${ }^{\circledR}$ NETWORK}

The GAITRite ${ }^{\circledR}$ system is a validated gait analysis system consisting of a portable electronic walkway, with software allowing immediate determination of spatiotemporal gait parameters (8-11). In order to create a discussion platform for European users of this system, the first European GAITRite ${ }^{\circledR}$ Meeting was held in Geneva in 2003. At this meeting, approximately fifty clinicians and researchers gathered to discuss data and present examples of clinical applications of spatio-temporal gait analysis. To enhance reproducibility of gait measures and for better comparability of outcomes, the participants reached a consensus on data collection guidelines that was officially approved by the network group in November 2004, at the $2^{\text {nd }}$ European GAITRite ${ }^{\circledR}$ Meeting in Marseilles. The guidelines listed below comprise the consensus, and are intended to facilitate network collaborations and provide guidance to clinicians who wish to implement spatiotemporal gait analysis in clinical settings.

\section{ENVIRONMENTAL MEASURE CONDITIONS AND SAFETY ISSUES \\ Light}

Measurements should be performed in a reproducible, well-lit environment. Indirect lighting is preferable, as are intensity-controlled artificial light and the capacity to control for changing day-light conditions by covering windows with black-out shades.

Key words: Falls, gait analysis, guidelines, older adults.

Correspondence: R.W. Kressig, MD, MOBEQ, Department of Rehabilitation and Geriatrics, Geneva University Hospitals, CH-1226 Geneva-Thonex, Switzerland.

E-mail: reto.kressig@hcuge.ch

Received December 3, 2004; accepted in revised form May 10, 2005. 


\section{Noise and visual distraction}

Data collection in a quiet, preferably closed room, with no auditive or visual interference for participants, is recommended.

\section{Clothing and footwear}

Clothing should be comfortable and non-restrictive. Participants should be allowed to walk in their own footwear. Appropriate types of footwear include closed walking shoes (no slippers), with heel height not exceeding 3 $\mathrm{cm}$ (12). For follow-up gait analyses, we highly recommend using the same footwear as was worn at baseline.

\section{Safety}

Depending on participants' fall risk, we recommend safety measures such as a safety-belt around the participant's waist for easy grabbing by an observer in case of an imminent fall. When using the GAITRite ${ }^{\circledR}$ system, it is preferable to fix the edges of the walkway to the floor with adhesive tape, to avoid slipping.

\section{MEASUREMENT PROCEDURES}

\section{Steady-state gait at different velocities}

We recommend measuring steady-state gait at different gait speeds, preferably in randomized order (i.e., slow, normal, fast), which allows for normalization of gait data for speed $(13,14)$. True steady-state gait can be observed by starting data collection after two complete gait cycles. For instance, this may be achieved by instructing participants to start walking at least 2 meters prior to reaching the electronic walkway and stopping at least 2 meters beyond it. If possible, we also recommend that all gait trials consist of walking in the same direction.

\section{Standardized walking instructions}

We suggest the use of simple, clear, standardized walking instructions to explain the various tasks to participants, i.e., "Please start walking at your usual speed towards the cone, now!", "Please start walking as fast as possible, without running or taking risks, towards the cone, now!" or, "Please start walking slow$\mathrm{ly}$, as if you were waiting for the bus, towards the cone, now!".

\section{Assistive devices}

We recommend allowing participants to use their own glasses and hearing aids, if worn. For the collection of network gait data (to be pooled in a common European data-base), we suggest either including only participants without assistive devices, or specifically describing the type of assistive device participants used in the study.

\section{Stride-to-stride variability}

In order to evaluate stride-to-stride variability, we recommend the highest possible number of gait cycles from a practical standpoint, with a minimum of three consecutive gait cycles for both left and right sides (i.e., a total of six gait cycles).

Gait analysis in association with simultaneous cognitive tasks

For dual-tasking in association with walking, we suggest commonly used and validated cognitive tasks such as counting backwards from 50 out loud, and a verbal fluency task such as enumerating as many animal names as possible $(4,15)$.

\section{Description of study population}

In studies of gait performance in older individuals, we suggest describing or categorizing older participants with a frailty classification (i.e., 16) and/or by their living condition (e.g., community-dwelling, sheltered housing, geriatric hospital, long-term care etc.).

\section{APPENDIX}

\section{Network group members}

Jennifer Anders, Hamburg, Germany, Olivier Beauchet, St. Etienne, France, Veronique Feipel, Brussels, Belgium, Ellen Freiberger, Erlangen, Germany, Thomas Fruehwald, Vienna, Austria, Claudine Geser, Zurich, Switzerland, Jorunn Helbostad, Trondheim, Norway, Inger Holm, Oslo, Norway, Michael Jamour, Ehingen, Germany, Reto W. Kressig, Geneva, Switzerland, Ulrich Lindemann, Stuttgart, Germany, Lillemor Lundin-Olsson, Umea, Sweden, Rolf Moe-Nilssen, Bergen, Norway, Katharina Pils, Vienna, Austria, Tischa Van der Cammen, Rotterdam, The Netherlands, Marianne Van Iersel, Nijmegen, The Netherlands.

\section{REFERENCES}

1. Kressig RW, Gregor RJ, Oliver A, et al. Temporal and spatial features of older adults transitioning to frailty. Gait Posture 2004; 20 : 30-5.

2. Maki BE. Gait changes in older adults: predictors of falls or indicators of fear. J Am Geriatr Soc 1997; 45: 313-20.

3. Hausdorff JM, Rios DA, Edelberg HK. Gait variability and fall risk in community-living older adults: a 1-year prospective study. Arch Phys Med Rehabil 2001; 82: 1050-6.

4. Beauchet O, Kressig RW, Najafi B, Dubost V, Mourey F. Age-related decline of gait control under a dual-task condition. J Am Geriatr Soc 2003; 51: 1187-8.

5. Sheridan PL, Solomont J, Kowall N, Hausdorff JM. Influence of executive function on locomotor function: divided attention increases gait variability in Alzheimer's disease. J Am Geriatr Soc 2003; 51: 1633-7.

6. Klenerman L, Dobbs RJ, Weller C, Leeman AL, Nicholson PW. Bringing gait analysis out of the laboratory and into the clinic. Age Ageing 1988; 17: 397-400.

7. Kressig RW, Beauchet O. Gait analysis and tailored exercise prescription in older adults. Z Gerontol Geriat 2004; 37: 15-9.

8. Menz HB, Latt MD, Tiedemann A, Mun San Kwan M, Lord SR. Reliability of the GAITRite system for the quantification of temporo-spatial parameters of gait in young and older people. Gait Posture 2004; 20: 20-5.

9. Bilney B, Morris M, Webster K. Concurrent related validity of the GAITRite walkway system for quantification of the spatial and temporal parameters of gait. Gait Posture 2003; 17: 68-74. 
10. McDonough AL, Batavia M, Chen FC, Kwon S, Ziai J. The validity and reliability of the GAITRite system's measurements: a preliminary evaluation. Arch Phys Med Rehabil 2001; 82: 419-25.

11. Cutlip RG, Mancinelli C, Huber F, DiPasquale J. Evaluation of an instrumented walkway for measurement of kinematic parameters of gait. Gait Posture 2000; 12: 134-8.

12. Lindemann U, Scheible S, Sturm E, et al. Elevated heels and adaptation to new shoes in frail elderly women. Z Gerontol Geriat 2003; 36: 29-34.

13. Helbostad JL, Moe-Nilssen R. The effect of gait speed on later- al balance control during walking in healthy elderly. Gait Posture 2003; 18: 27-36.

14. Moe-Nilssen R, Helbostad JL. Interstride trunk acceleration variability but not step width variability can differentiate between fit and frail older adults. Gait Posture 2005; 21: 164-70.

15. Beauchet O, Dubost V, Gonthier R, Kressig RW. Dual-task-related gait changes in transitional frail older adults: the type of the walkingassociated cognitive task matters. Gerontology 2005; 51: 48-52.

16. Fried LP, Tangen CM, Walston J, et al., Cardiovascular Health Study Collaborative Research Group. Frailty in older adults: evidence for a phenotype. J Gerontol 2001; 56: M146-56. 